\title{
Liquid crystal suspensions of carbon nanotubes assisted by organically modified Laponite nanoplatelets
}

\author{
O. Yaroshchuk ${ }^{a, b, *}$, S. Tomylko ${ }^{b}$, O. Kovalchuk ${ }^{b}$, N. Lebouka $^{c}$ \\ a Liquid Crystal Institute, Kent State University, 1425 University Esplanade, Kent, OH 44242-0001, USA \\ ${ }^{\mathrm{b}}$ Institute of Physics, NAS of Ukraine, Prospekt Nauky 46, 03028 Kyiv, Ukraine \\ ${ }^{c}$ Institute of Biocolloidal Chemistry named after F.D. Oucharenko, NAS of Ukraine, 42, blur. Vernadskogo, 03142 Kyiv, Ukraine
}

\section{A R T I C L E I N F O}

Article history:

Received 10 June 2013

Accepted 6 November 2013

Available online 15 November 2013

\begin{abstract}
A B S T R A C T
We present a simple and effective approach for dispersion of carbon nanotubes (CNTs) into liquid crystals (LCs), suitable for a wide range of CNT concentrations ( $\leqslant 0.1 \mathrm{wt} . \%)$. It consists in doping of LC+CNT suspensions with Laponite, which shows high affinity to the nanotubes. The organo-modified Laponite platelets surround CNTs and hamper their aggregation. At the same time, they do not hinder orientational ordering of CNTs imposed by LC matrix. This approach avoids problems of direct organo-modification of CNTs, associated with worsening of their unique properties. The increased dispersion degree results in drastic changes in dielectric and electro-optical characteristics of the suspensions. Dispersing and insulating effects of the Laponite platelets on CNTs lead to the absence of classical percolation of conductivity, linear growth of dielectric constant with CNT concentration, as well as reduction of Frederiks threshold and significant growth of contrast ratio of the E7+CNTs suspensions.
\end{abstract}

(c) 2013 Elsevier Ltd. All rights reserved.

\section{Introduction}

Enormous interest to carbon nanotubes (CNTs), observed over the last two decades, is mainly caused by large anisotropy of the physical properties of these objects associated with extraordinarily large anisotropy of their shape (the lengthto-diameter ratio of the tubes is, typically, 500-1000). CNTs are characterized by high mechanical strength in the tube axis combined with flexibility in transversal direction, and large anisotropy of electric and thermal conductivity, reaching values typical for metals or semiconductors in the axial direction. Besides, CNTs demonstrate high chemical resistance. Due to these properties, CNTs are distinguished as advanced materials for various potential applications, such as energy storage and conversion, displays, nano-scale electronics, nano-sensors for chemical and biological molecules, artificial muscles, etc. [1,2].

For many of these applications the nanotubes are required to be aligned. In particular, the alignment of CNTs is a key issue in nanoelectronics, field emitters and sensors. The alignment can be achieved by directional growth of nanotubes [3] or orienting action on the ensembles of synthesized tubes with the help of external fields (electric or magnetic field, mechanical stress, etc.) [4,5]. The other approach exploits ability of anisotropic nanoobjects spontaneously organize into liquid crystalline ordering, being in a colloidal state 6-8]. However, the research aimed at the large scale alignment of nanotubes using these methods is in its infancy.

\footnotetext{
* Corresponding author at: Liquid Crystal Institute, Kent State University, P.O. BOX 5190, 1425 University Esplanade, Kent, OH 44242-0001, USA. Fax: +1 3306722796 .

E-mail addresses: o.yaroshchuk@gmail.com (O. Yaroshchuk), tomulkosv@ukr.net (S. Tomylko), akoval@knutd.com.ua (O. Kovalchuk), lebovka@gmail.com (N. Lebovka).
} 0008-6223/\$ - see front matter (c) 2013 Elsevier Ltd. All rights reserved. http://dx.doi.org/10.1016/j.carbon.2013.11.015 
Essentially other idea, which is recently actively explored, is to disperse CNTs in anisotropic organic matrixes. Different self-assembling materials, such as surfactants, block copolymers, and liquid crystals (LCs), have been tested as organic hosts for CNTs. Among them, the LC hosts characterized by long-range orientational order show special promise $[9,10]$. They allow one to attain oriented ensembles of nanotubes of macroscopic scale. This alignment can be patterned and driven by external fields. Finally, by removing the LC, orientationally ordered structures of pure CNTs can be obtained [11].

The other reason for interest in LC+CNT suspensions is the possibility to improve dielectric characteristics and electrooptic response of LC layers in displays and other devices; reduction of switching voltage [12] and response time [13], elimination of image sticking [14] and back flows [15] have been described in literature. These results give an interesting hint to further improvement of LC electro-optic devices [16].

The problem that hinders attaining of the mentioned benefits is insufficient dispersion of CNTs. Giant aspect ratio along with high flexibility of CNTs leads to a high degree of their entanglement. Due to great length $(\sim 1-10 \mu \mathrm{m})$ of CNTs, the integral interaction energy of two neighboring CNTs is extremely large $\left(E_{\mathrm{CNT}-\mathrm{CNT}} \sim 10^{3} \mathrm{eV}\right)$, despite the fact that nanotubes interact via weak van der Waals forces. Because of these factors, CNTs bundle and cluster together in disordered agglomerates. It drastically deteriorates the beneficial effects anticipated from single nanotubes. Agglomeration weakens anisotropic properties of CNTs, their integration in LC matrices and optical uniformity of LC layers. Thus, orientational ordering of CNTs and actual improvement of properties of LC layers after their doping by CNTs become possible if the problem of strong aggregation of the nanotubes is overcome.

The approaches to improvement of dispersing of CNTs in LC hosts known today can be conventionally divided into physical and chemical. The physical approaches include optimization of LC materials and mixing conditions [17], in situ electro-hydrodynamic (EHD) dispergation [18,19] and debundling of CNTs in high electric fields [20]. The chemical approaches involve chemical attaching of various fragments to CNTs via covalent bonding [21], $\pi-\pi$ stacking interaction [22], etc. All of these approaches have certain limitations. For example, the EHD dispergation is applicable only to LC+CNT suspensions, where intensive EHD flows can be initiated. The nanotube dissociation in a high electric field carries high risk of electric breakdown. In turn, chemical modification of the surface of CNTs affects the extended $\pi$ systems, responsible for the unique properties of these particles. Thus, despite the variety of approaches, the problem of obtaining highly dispersed and stable suspensions of CNTs in LC is not completely resolved and remains topical.

In this work, we have applied an essentially new approach. It implies assistance of CNT dispersion by nanoparticles of another type. This principle has been known for more than 10 years, since Tohver et al. observed the remarkable stabilizing effect of highly charged nanoparticles on colloidal spheres dispersed in water [23]. The nanoparticles formed a kind of halo around the spheres (haloing effect) preventing their coagulation. This approach proved to be quite effective for CNT suspensions. The stabilizing agents, most frequently used for nanotubes, are inorganic platelets (IP) showing high affinity to CNTs. In particular, it was shown that CNTs can be easily deroped in an aqueous solution by addition of $\mathrm{ZrP}$ [24] or Laponite [25] nanoplatelets. The stabilizing effect of IP was also clearly observed for polymer suspensions of CNTs [24,26-29]. In many cases, combination of CNTs and IP resulted in a synergistic effect on mechanical, electrical, flame retardation and other properties of polymers.

The aim of our research is to adapt this approach to stabilization of nanotubes in a liquid crystal. Previously, we tried to achieve this by doping the LC+CNT suspensions with organically modified particles of natural clays (montmorillonite (MMT)). However, the results appeared quite ambiguous and highly dependent on the origin of the clay minerals [30]. In the present research, we apply a synthetic clay Laponite (Lap) as a stabilizing agent, mainly because of two reasons. Firstly, the synthetic particles have identical chemical content and structure, which are well controlled in the course of synthesis. Secondly, the size of Laponite platelets is much smaller than the size of MMT platelets ( $\sim 25 \mathrm{~nm}$ vs. $\sim 10 \mu \mathrm{m}$ ), and thus is comparable to the transversal dimension of nanotubes. We demonstrate that dispergation of CNTs in a nematic LC highly improves in the presence of a small amount of organically modified Laponite that radically changes the dielectric and electro-optical properties of E7+CNT suspensions.

\section{Experimental}

\subsection{Materials}

The dispersing medium in this research was LC E7 from Merck, as a well characterized and the most frequently used LC material in the study of LC-CNT composites. It is a eutectic mixture of three cyanobiphenyl and one cyanotriphenyl compounds with the nematic-to-isotropic transition at $58^{\circ} \mathrm{C}$. At room temperature, the dielectric constants of this mixture in the directions parallel and perpendicular to the LC director are $\epsilon_{/ /}=19$ and $\epsilon_{\perp}=5.2$, respectively. As CNTs we utilized the multi-walled carbon nanotubes from Spetsmash Ltd. (Kyiv, Ukraine), produced from ethylene by the chemical vapor deposition method [31]. Typically, these CNTs had an outer diameter $20-40 \mathrm{~nm}$, while their length ranged from 5 to $10 \mu \mathrm{m}$. The specific electric conductivity $\sigma$ of the powder of compressed CNTs was about $10 \mathrm{~S} \mathrm{~cm}^{-1}$. The synthetic clay used in this work was Laponite from Rockwood Additives Ltd., U.K. Its formula is $\mathrm{Na}_{0.7}\left[\left(\mathrm{Si}_{8} \mathrm{Mg}_{5.5}\right.\right.$ $\left.\left.\mathrm{Li}_{0.4}\right) \mathrm{O}_{20}(\mathrm{OH})_{4}\right]$. It is a powder, in which the disc-like nanoplates of the clay are packed into stacks. The thickness and diameter of these discs are about $1 \mathrm{~nm}$ and $25-30 \mathrm{~nm}$, respectively. The faces of disks have a constant negative charge, while the surface charge of their edges is $\mathrm{pH}-$ dependent and positive in acidic medium [32]. As described elsewhere [29], the platelets of original Laponite were modified by ion-exchange reactions with the surfactant cetyl-trimethylammonium-bromide (CTAB, $\mathrm{C}_{16} \mathrm{H}_{33}-\mathrm{N}\left(\mathrm{CH}_{3}\right)_{3} \mathrm{Br}$, Fluka, Germany) with $99.5 \%$ purity. The resultant material will be further called the organomodified Laponite (LapO). 


\subsection{Preparation of samples}

LCs filled with CNTs, LapO or their hybrid mixtures were obtained by adding appropriate weights of CNTs ( $C=0.025-0.5 \mathrm{wt} . \%$ ) and LapO (0.1 wt.\%) to E7 at T $=60^{\circ} \mathrm{C}$ with subsequent $10 \mathrm{~min}$ sonication using a sonotrode ultrasonic disperser at $22 \mathrm{kHz}$ and $250 \mathrm{~W}$. Then suspensions were incubated at room temperature for $24 \mathrm{~h}$, sonicated again for 2 min and loaded by capillary forces into the cells. The loaded particles practically did not affect the temperature $T_{c}$ of the nematic-isotropic phase transition of E7 $\left(\Delta \mathrm{T}_{c}<0.2^{\circ}\right)$.

The cells for electro-optical and most of dielectric measurements were made from glass substrates, containing patterned ITO electrodes and aligning layers of polyimide AL3046 (JSR, Japan) for planar alignment. To determine anisotropy of dielectric constant, the substrates for homeotropic alignment were additionally prepared. They contained the layer of polyimide SE1211 (Nissan, Japan). The polyimide layers were obtained by spin coating technique, properly backed and rubbed by a fleecy cloth in order to provide a uniform planar alignment of LC in the field-off state. The cells were assembled so that the rubbing directions of the opposite aligning layers were antiparallel or perpendicular, thus the antiparallel or twisted cells were obtained. The antiparallel cells were further used in dielectric studies, while the twisted cells were used for electro-optic measurements. The cell gap $d$ was maintained by $20 \mu \mathrm{m}$ glass spacers.

\subsection{Methods}

The alignment uniformity in the cells was checked using a light box equipped with two polarizers. The microstructure of the samples was studied using the optical polarization microscope Polam L-213 M, equipped by digital camera conjugated with personal computer. The black and white microscopic images were analyzed using Hoshen-Kopelman algorithm [33] to determine the mean radius of aggregates $r_{m}$ and their size distribution $f(r)$.

The dielectric studies were conducted by oscilloscopic method [34]. The resistance and capacitance of the LC cells were experimentally measured in a wide frequency range, $f=10^{-1}-10^{6} \mathrm{~Hz}$, and used for calculation of real $\varepsilon^{\prime}$ and imaginary $\varepsilon^{\prime \prime}$ parts of complex dielectric constant $\varepsilon^{*}=\varepsilon^{\prime}-i \varepsilon^{\prime \prime}$. The frequency dependences of $\varepsilon^{\prime}$ and $\varepsilon^{\prime \prime}$ were plotted and the frequency range $10<f<10^{5} \mathrm{~Hz}$ free of any relaxation processes was selected for further evaluation of permittivity $\varepsilon^{\prime}$ and conductivity $\sigma$ values corresponding to the volume part of samples. Defining values of a dielectric constant in homeotropically and planar aligned samples, respectively $\epsilon_{\|}^{\prime}$ and $\epsilon_{\perp}^{\prime}$, the value of dielectric anisotropy $\Delta \epsilon^{\prime}=\epsilon_{\| \prime}^{\prime}-\epsilon_{\perp}^{\prime}$ was obtained. The AC conductivity $\sigma$ was estimated from the formula $\sigma=2 \pi 2 \varepsilon_{0} \varepsilon^{\prime \prime} f$, where $\varepsilon_{0}$ is the electric permittivity of free space.

The electro-optical measurements were carried out using the setup described in [35]. In these experiments, the transmittance $T$, of the twist cells, placed between two parallel polarizers, was measured as a function of the applied AC voltage $U(f=2 \mathrm{kHz})$ ramped up from 0 to $30 \mathrm{~V}$. The cells were operated in a waveguide regime (the Mauguin's regime) [36], so that polarization of the testing light followed the LC director, which experienced rotation in $90^{\circ}$. The electro-optic contrast CR was calculated on the basis of $T(U)$ curve, according to formula $C R=T_{s} / T_{0}$, where $T_{0}$ and $T_{s}$ are the transmittance values in the initial and saturated states, respectively. Also, the threshold voltage of electric switching (Frederiks's threshold) was estimated as the voltage corresponding to the transmittance value $T_{0}+0.1\left(T_{s}-T_{0}\right)$.

The alignment of CNTs in the LC matrix was analyzed by measuring polarized Raman spectra of the suspensions. The spectra were recorded in the range $100-3000 \mathrm{~cm}^{-1}$ by using Renishaw inVia micro-Raman spectrometer. The measurements were carried out at room temperature in a backscattering geometry by sample excitation at $632.8 \mathrm{~nm}$ with $\mathrm{He}-\mathrm{Ne}$ laser. The laser beam was focused on the suspension layer by using long-focus objective $\times 20$. Two cases were analyzed: when polarizations of both waves were parallel to the director of LC (pp geometry) and when both polarizations were perpendicular to the director (oo geometry).

\section{Results and discussion}

\subsection{Microscopic structure}

At the beginning, Laponite was optimized for its effective dispersion in LC. In agreement with [37], we observed that neat Laponite poorly exfoliates in nematic LCs and exists in a form of pretty large grains (tens of microns by size). These grains effectively aggregate, grow and percolate with increasing Lap concentration. To promote exfoliation of Laponite stacks and stabilize the Lap platelets formed, the Laponite was modified with ionic surfactant CTAB. The obtained organo-modified Laponite (LapO) dispersed very well in nematic LCs up to concentration of $0.2 \mathrm{wt}$ \% (Fig. S1).

This LapO sample was further used to prepare ternary mixtures LC+CNT+LapO, based on nematic LC E7 from Merck, different amounts of CNT (0.025-0.5 wt.\%) and fixed amount of LapO (0.1 wt.\%). As reference samples, we studied E7+CNT (0.025-0.5 wt.\%) and E7+LapO (0.1 wt.\%) suspensions and pure LC E7. The mixtures were filled in the LC cells with planar anchoring, provided by rubbed PI layers. Generally, the planar alignment was maintained in all samples. The E7+LapO samples demonstrated high alignment uniformity comparable with pure E7 samples and no essential Lapo particle aggregates. However, the alignment uniformity worsened in E7+CNT and E7+CNT+LapO suspensions due to director perturbation in the vicinity of nanoparticle aggregates. This worsening was enhanced with concentration of CNTs due to increasing size and number of CNT aggregates (Fig. 1).

The size distributions and mean radii of CNT aggregates, determined by cluster analysis of the images of Fig. 1, are presented in Fig. 2.

According to Fig. 2, increase in CNT concentration results in broadening of the size distribution of CNT aggregates and growth of the mean size of aggregates in the transversal direction. The latter process is especially pronounced for the E7+CNT series. In this case, the steep growth starts at about $C=0.1$ wt. $\%$ where the size of aggregates is comparable 
(a)
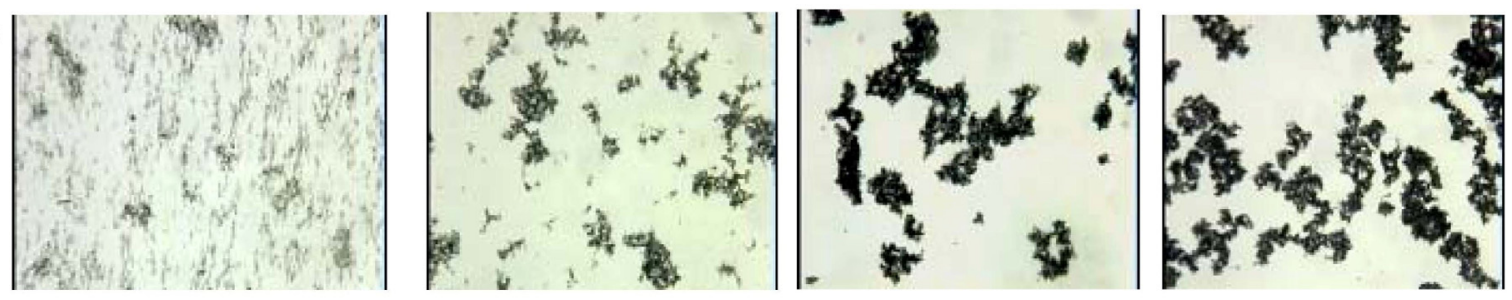

(b)

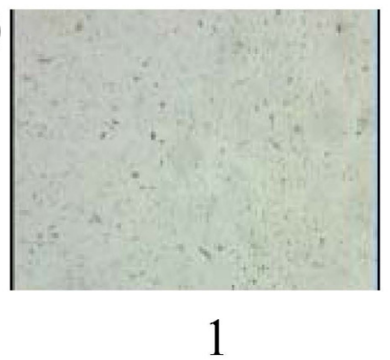

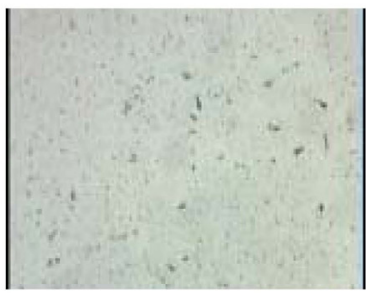

2

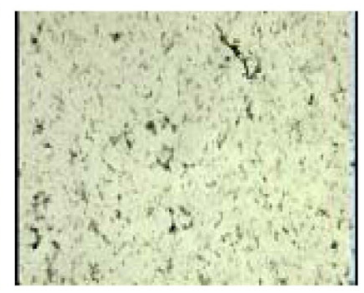

3

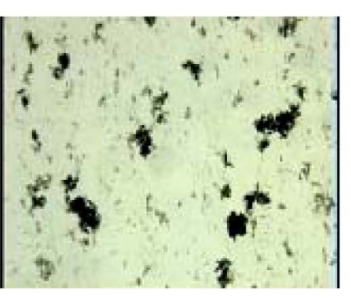

4

Fig. 1 - Microscopic pictures of the layers of (a) E7+CNT and (b) E7+CNT+LapO (0.1 wt.\%) suspensions. Concentration of CNTs in the samples 1, 2, 3 and 4 is $\mathbf{0 . 0 2 5}, \mathbf{0 . 0 5}, \mathbf{0 . 1}$, and $0.3 \mathrm{wt}$.\%, respectively. (A colour version of this figure can be viewed online.)

to the cell thickness $d=20 \mu \mathrm{m}$ and reaches hundreds of microns at $C \geqslant 0.3$ wt.\%. Finally, at $C>0.3 \%$, the bulky aggregates overlap forming the geometrical percolation network that spans throughout the sample.

For the E7+CNT+LapO series the structural evolution is different. In this case, the size of CNT aggregates becomes essential only at $C \geqslant 0.1 \mathrm{wt} . \%$, but the network structure of the aggregates is not formed even at $C=0.3 \mathrm{wt}$ \% (Fig. 1). The difference in the structure of E7+CNTs and E7+CNTs+LapO suspensions is particularly striking in the range of $C<0.05 \mathrm{wt} . \%$, where CNTs in the LapO-assisted suspensions are practically in disaggregated form. It should be noted that the value $0.05 \mathrm{wt} . \%$ is about one order of magnitude higher than the value of $C$ typical for the diluted E7+CNTs suspensions commonly used by researchers. Consequently, this result suggests a promising way to stabilize CNTs in a LC, which is

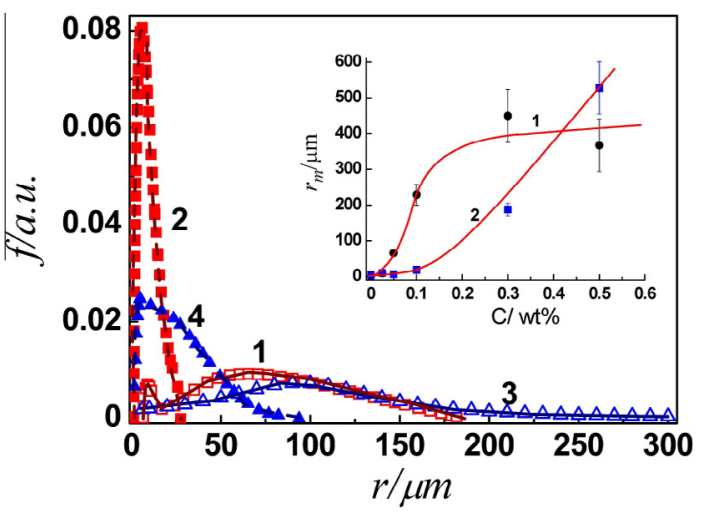

Fig. 2 - (a) The size distribution functions of CNT aggregates for E7+CNT and E7+CNT+LapO suspensions with different proportions of CNTs and LapO: (1) $0.05 \mathrm{wt}$ \% CNT, (2) 0.05 wt.\% CNT+0.1 wt.\% LapO, (3) 0.1 wt.\% CNT, and (4) $0.1 \mathrm{wt} . \% \mathrm{CNT}+0.1 \mathrm{wt} . \%$ LapO. Inset shows the mean radius of the CNT aggregates $r_{m}$ versus concentration of CNTs, C. (A colour version of this figure can be viewed online.) efficient for rather broad concentration range of the nanotubes.

Strong disintegration of CNTs in the presence of LapO platelets is easily explained in terms of the haloing effect. This effect is realized in our system because the intermediate LapO particles exhibit high affinity for both components of LC-CNT suspensions. Good affinity of organically modified clay particles for LC leads to the fact that the stacks of Laponite effectively exfoliate in LC releasing big numbers of nanoplatelets [38]. A good affinity of LapO for CNTs implies strong interactions between these particles having approximately the same diameter [25]. Although the corresponding microscopic mechanisms are not clear yet, it can be assumed that the energy of this interaction is much higher than the energy of the weak van der Waals interaction of nanotubes. Because

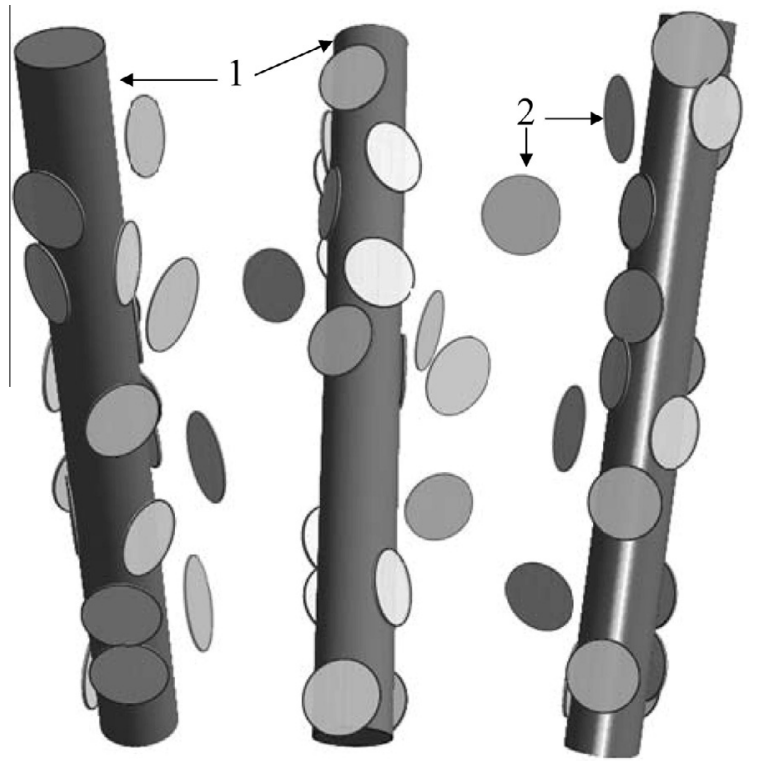

Fig. 3 - Schematic representation of the structure of CNT (1) and LapO (2) particles in LC host. 
of this, LapO platelets surround the nanotubes destroying bulky aggregates and splitting the nanotube bundles. The resulting structure is illustrated in Fig. 3.

It is worthwhile mentioning limitations of the considered approach. According to Fig. 2, at $\mathrm{C}=0.5 \mathrm{wt} . \%$, the sizes of aggregates in the $\mathrm{E} 7+\mathrm{CNT}$ and $\mathrm{E} 7+\mathrm{CNT}+\mathrm{LapO}$ suspensions become comparable, providing evidence for a weaker stabilizing effect of LapO. In supplementary studies, we tried to enhance the stabilizing effect of LapO by increasing its concentration to $0.5 \mathrm{wt} . \%$. However, we did not observe substantial strengthening of disaggregation of CNTs at high concentrations of LapO ( $C \geqslant 0.5 \mathrm{wt}$ \%). Perhaps, this is due to supersaturation of LC with LapO particles that restricts their complete exfoliation. It is supported by observation of LapO stacks aggregating at this concentration (Fig. S1).

Finally, consider temporal stability of the samples. In accordance with previous studies [39], aging of the E7+CNT samples frequently resulted in gradual grow of the aggregates of CNTs and changing of electro-optic response. Long-term experiments with E7+CNTs+LapO suspensions did not reveal such changes at least over 3 months period.

\subsection{Dielectric properties}

In the following we clarified manifestation of the improved dispersion of CNTs in physical properties of the suspensions. In dielectric studies, we analyzed dielectric spectra of the samples focusing mainly on the frequency range corresponding to the processes of polarization and conduction in the bulk of the sample (range B in Fig. S6). In this range, there are no relaxation processes, thus the dielectric constant $\varepsilon^{\prime}$ and the electrical conductivity $\sigma$ are independent of the frequency.

The $\sigma(C)$ curves for the E7-CNT and E7-CNT-LapO composites are presented in Fig. 4. It is evident that the effect of CNTs on the conductivity of E7 and E7+LapO (0.1 wt.\%) samples is essentially different. In the case of E7, increasing CNT concentration results in a sharp increase of conductivity in more than one order of magnitude and, subsequently, its saturation. Qualitatively, this behavior agrees well with that established earlier for the LC-CNT systems [35]. It is typical for electrical percolation to be described by a scaling equation [40]

$\sigma=a\left(c-c_{c}\right)^{t}$

where $c_{c}$ is the critical concentration and $t$ is the transport index. The $\sigma(C)$ curves for E7-CNT series obey this law very well (inset in Fig. 4a). The least-square fitting of these curves to the equation (1) yields $C_{c} \leqslant 0.003$ wt. \% and $t=0.5-0.6$.

First of all, note that $\sigma(c)$ dependences are essentially sublinear $(t<1)$. The obtained values of $t$ are smaller than theoretical values for $3 d(t=2[40])$ and even $2 d(t=4 / 3[41,42])$ random percolation, which is more probable for our case. This may be caused by limitations of general percolation theory neglecting orientational order typical for LCs. Besides, lowering of the transport index $t$ can be stipulated by solvation of CNTs with polar molecules of LC E7. This process affects electrical contacts between the nanotubes and thus hinders their percolation. As shown in Fig. 1, at moderate concentrations of CNTs, the nanotube aggregates are rather loose and consist of
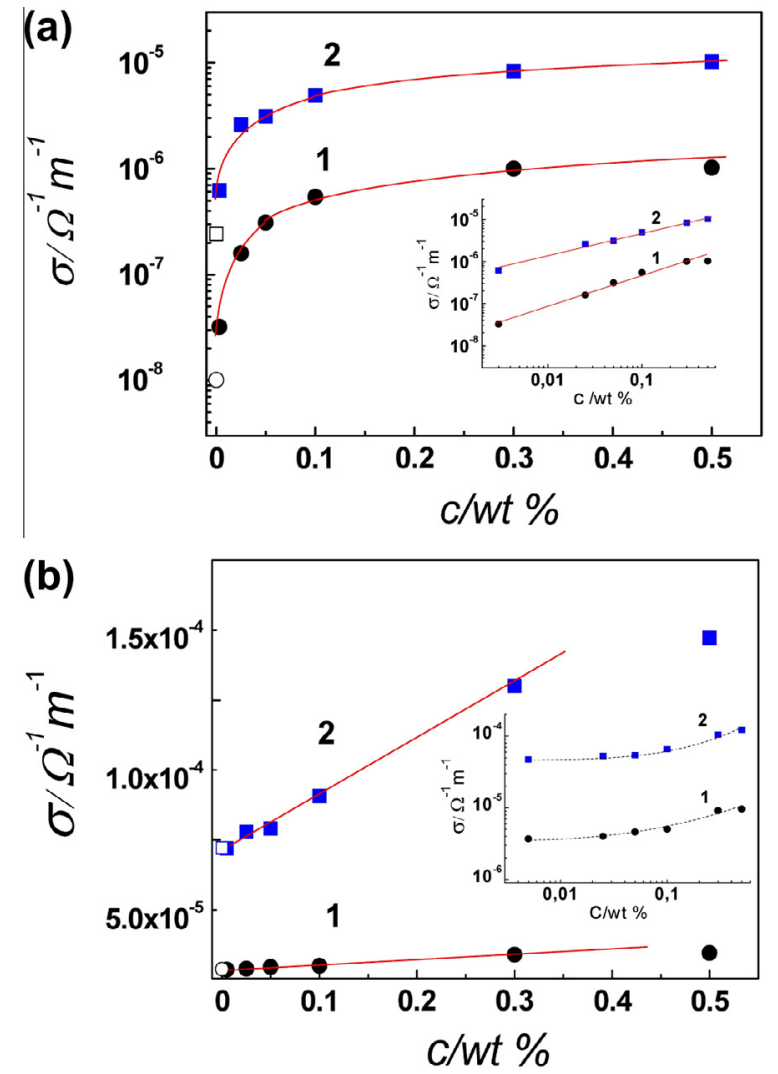

Fig. 4 - Measured (symbols) and fitted (lines) conductivity vs. CNT concentration curves for (a) E7-CNT and (b) E7-CNTLapO series, corresponding to temperature of $20^{\circ} \mathrm{C}$ (curve 1) and $80^{\circ} \mathrm{C}$ (curve 2). The insets show the same curves in double logarithmic scale to identify the percolation law (1). In this case, the data corresponding to $C=0$ (unfilled symbols) are excluded from fitting process. It can be seen that the $\sigma(C)$ curve for E7-CNT series is fitted well to the power law (1), while the curve for the E7-CNT-LapO series is essentially linear up to $C=0.3 \mathrm{wt}$.\%. (A colour version of this figure can be viewed online.)

smaller formations surrounded by LC. Increasing of $C$ leads to denser packing of CNTs that reduces the distance between the nanotubes and thus improves the number and the quality of electrical contacts between them. Owing to this, the percolation of conductivity lags behind the geometrical (visible) percolation at increasing concentrations of nanotubes. Solvation strengthens the role of the ionic mechanism of conductivity, typical for a LC host, characterized by sublinear dependence of conductivity on ion concentration [36]. The ion sources in our samples are nanoparticles. Really, the conductivity of E7 jumps with the introduction of a minute amount of CNTs and/or LapO due to an increase in the concentration of ions, which desorb from the surface of the particles.

A very small value of the critical concentration $C_{c}$ is due to the small distance between the electrodes $(d=20 \mu \mathrm{m})$, which is comparable to the length of the CNTs $(5-10 \mu \mathrm{m})$. It means that the channels of high conductivity, associated with the CNTs (individual CNTs and their aggregates), start to form in the direction perpendicular to the composite layer at a very 
low concentration of CNTs. In turn, the geometrical network in the plane of the composite layer, which is visible in polarizing microscope (Fig. 1), gets formed at considerably higher concentration of CNTs, $C_{c}>0.1 \mathrm{wt} . \%$. This is due to the fact that the size of the aggregates in the plane of the layer is practically unlimited, in contrast to the perpendicular direction, where the size is limited by the thickness of the cell. Thus, the aggregates formed in our cells are largely two-dimensional. One can also conclude that electric conductivity and optical microscopy data reflect percolation of CNTs in different directions.

In big contrast to $\mathrm{E7}+\mathrm{CNT}$ samples, the $\sigma(\mathrm{c})$ curves for $\mathrm{E} 7+\mathrm{CNT}+\mathrm{LapO}$ series do not obey the percolation low (1). Instead, they are essentially linear up to $C=0.3 \mathrm{wt}$ \% (Fig. $4 b$ ). In view of haloing effect, elimination of conductivity percolation is caused by low rate of aggregation of CNTs. Moreover, the dielectric platelets of LapO surrounding nanotubes prevent electrical contacts between them. In this situation, ionic conductivity mechanism, characterized by linear dependence of conductivity on ion concentration, becomes dominating.

It should be noted that such character of $\sigma(c)$ curves in E7CNT-LapO composites was observed against the background of sharply increased conductivity (by about two orders of magnitude compared with pure LC). The reasons for this are being studied. But tentatively we believe that this is primarily due to water absorbed by Laponite, which is mainly trapped between the platelets in the stacks [43]. This water can not be effectively removed by a deep freezing in vacuum, used in our research as a drying method [29]. The matter is the conventional high temperature drying can not be applied to LapO powders, because of risk of structural changes. During exfoliation of LapO stacks the trapped water is released in LC. According to [44], the dissolved water dissociates and promotes dissociation of other impurities that is a main factor of increasing of ionic conductivity. The other factor could be the charge migration along the surface of the network of LapO particles, which may be quite essential at LapO concentration of 0.1 wt.\% [30].

The $\varepsilon^{\prime}(c)$ dependences are shown in Fig. 5. It can be seen that $\varepsilon^{\prime}$ monotonically grows with $C$ and increases by almost two times with addition of $0.5 \mathrm{wt} . \%$ of CNTs. This trend is consistent with the earlier results indicating the increase of LC permittivity with the addition of a minute amount of CNTs [45]. The growth of $\varepsilon^{\prime}$ was observed in both nematic and isotropic phases indicating that it is mainly due to the contribution of the polarizability of nanotubes rather than disturbance of LC orientation by the particles and their aggregates. The $\varepsilon^{\prime}(C)$ curve for the E7-CNT series can be satisfactorily fitted to the percolation law (1) that supports collective polarization mode of nanotubes at $C>C_{c}$.

In contrast, the $\varepsilon^{\prime}(C)$ curve for the E7-CNT-LapO series (Fig. 5b) in the studied range of CNT concentrations is linear, which indicates that this series is far from percolation. In view of this fact, the $\varepsilon^{\prime}(C)$ curve can be fitted to Maxwell-Wagner mixing equation:

$\epsilon^{\prime}=\epsilon_{\mathrm{LC}}^{\prime}+\mathrm{KC}$

derived in the $C<<1$ approximation [46]. Here, $K$ is a constant combining permittivity of LC, $\varepsilon_{\mathrm{LC}}^{\prime}$, and CNT, $\varepsilon_{\mathrm{CNT}}^{\prime}$. Formula (2)
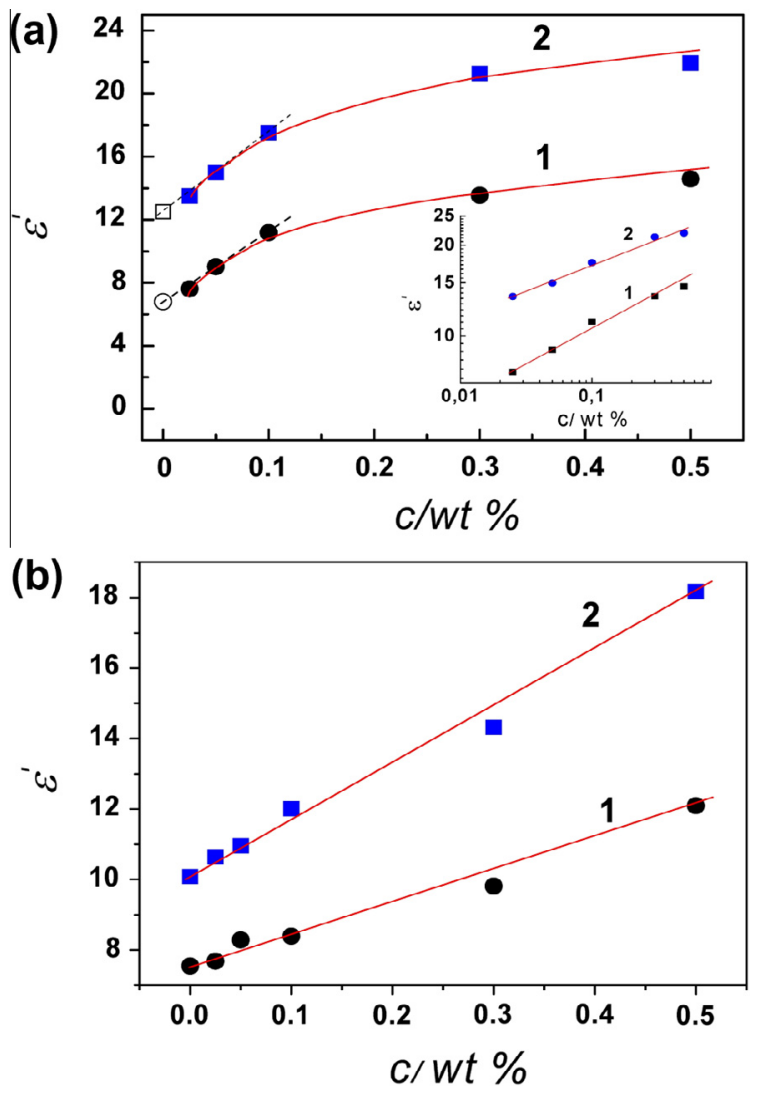

Fig. 5 - Measured (symbols) and fitted (lines) permittivity vs. CNT concentration curves for (a) E7-CNTs and (b) E7-CNTLapO series, corresponding to temperature $20^{\circ} \mathrm{C}$ (curve 1) and $80^{\circ} \mathrm{C}$ (curve 2). The insets in case (a) shows the same curves in double logarithmic scale to demonstrate the percolation law (1). In this case, the data corresponding to $C=0$ (unfilled symbols) are excluded from the fitting process. It can be seen that the $\varepsilon^{\prime}(C)$ curve for E7-CNT series can be satisfactorily fitted to the power law (1); $C \leqslant 0.003$, $p=0.16-0.21$. In contrast, the linear fitting is appropriate in the case (b). (A colour version of this figure can be viewed online.)

predicts linear dependence of $\varepsilon^{\prime}$ on $C$, which is in full accordance with the result for the E7-CNT-LapO series. In turn, the $\varepsilon^{\prime}(C)$ curves for the E7-CNT series, where the percolation point is quickly achieved, can be satisfactorily fitted to a linear function only at their initial growing stage $(C<0.1 \mathrm{wt} . \%$, Fig. 5a).

Comparing data for dielectric constant in the cells with planar and homeotropic anchoring, the dielectric anisotropy $\Delta \varepsilon^{\prime}$ was estimated. Fig. S7 shows that $\Delta \varepsilon^{\prime}$ increases with concentration of CNTs that indicates partial orientational ordering of the tubes in LC [9]. The same trend is observed for the samples containing Laponite. This conclusion is consistent with the formation of anisotropic aggregates and their partial ordering at a low concentration of nanotubes (Fig. 1a, picture 1). The discrepancy observed at high concentrations of nanotubes is apparently due to the fact that, unlike the large aggregates visible in microscope, small aggregates and individual nanotubes are well integrated in LC order. 
Finally we note that the addition of Laponite also changes the dielectric properties at low frequencies, determined by the near-electrode layers. For the sake of brevity, we do not consider here these effects in detail. Yet, we note that, as in the bulk of the composites, the addition of Laponite leads to a weakening of percolation of CNTs, resulting in a moderate decrease in the efficiency of shunting of the near-electrode layers with the tubes [35], as well as in accelerating the near-electrode dielectric relaxation (Fig. S8).

\subsection{Raman spectroscopy studies}

As one of the most popular techniques of CNTs characterization, Raman spectroscopy was involved for independent verification of the orientational ordering of the nanotubes in the LC matrix $[47,48]$. The measured spectra of CNTs and LC E7 are shown, respectively in Fig. $6 a$ and b. The spectrum of LC E7 demonstrates four pronounced picks, which are practically identical to the picks of LC 5CB, main component of the nematic mixture E7 [47]. The most intensive band is at $1606 \mathrm{~cm}^{-1}$ and corresponds to $\mathrm{C}-\mathrm{C}$ stretch of aromatic rings. The other three bands centered at 1180,1282 and $2228 \mathrm{~cm}^{-1}$ correspond to $\mathrm{C}-\mathrm{H}$ in-plane deformation, $\mathrm{C}-\mathrm{C}$ stretching of biphenyl link and $\mathrm{C} \equiv \mathrm{N}$ vibrations.

In the spectrum of nanotubes three intense bands are detected. G band at $1578 \mathrm{~cm}^{-1}$ is typical for graphite-like materials and characterizes planar vibrations of carbon atoms in $\mathrm{sp}^{2}$ sites. The band $\mathrm{G}^{\prime}$ centered at $2650 \mathrm{~cm}^{-1}$ is indicative for graphene structures, and the band $\mathrm{D}$ at $1330 \mathrm{~cm}^{-1}$ is associated with some disorder to graphene structure, irregularities between the walls of multi-walled CNTs and inside the aggregated CNT bundles [49]. Characteristic peaks of LapO are not discussed here, since they do not appear in the Raman spectra of the LC+CNT+LapO composites (Fig. S9).

The problem of detecting of CNTs in the composites was that the Raman bands of the tubes well overlapped with the bands of LC E7 (Fig. 6). To enhance signal from CNTs, their concentration in the composites was set at $0.1 \mathrm{wt} . \%$ that corresponded to maximal value for which effective dispergation was detected. Besides, thickness of LC cells was increased to $50 \mu \mathrm{m}$.

Raman spectra of the E7+CNT+LapO composite measured in the ee and oo geometries are presented in Fig. $6 \mathrm{c}$. In these spectra, along with the intense bands of E7, weak reflexes of CNTs can be detected. The most pronounced of them is the band $\mathrm{D}$ at $1330 \mathrm{~cm}^{-1}$, which is fortunately not shaded by the neighboring band at $1282 \mathrm{~cm}^{-1}$ related to LC host. It is worthwhile mentioning that one cannot see clearly the band $G$, used in [47] for detecting the alignment of CNTs. Apparently, this is due to different intensity of this band for single-walled CNTs [47] and multi-walled CNTs used in our study. In this regard, the band D was selected as indicator of alignment of CNTs. One can see that along with the spectral bands corresponding to E7, the band D shows a pronounced dichroism. Similar result was obtained for the composites without LapO particles. This additionally confirms orientational ordering of CNTs in the LC+CNT and LC+CNT+LapO composites, earlier detected in dielectric studies. At the same time, comparison of Raman spectra for pure LC and the LC loaded with CNT
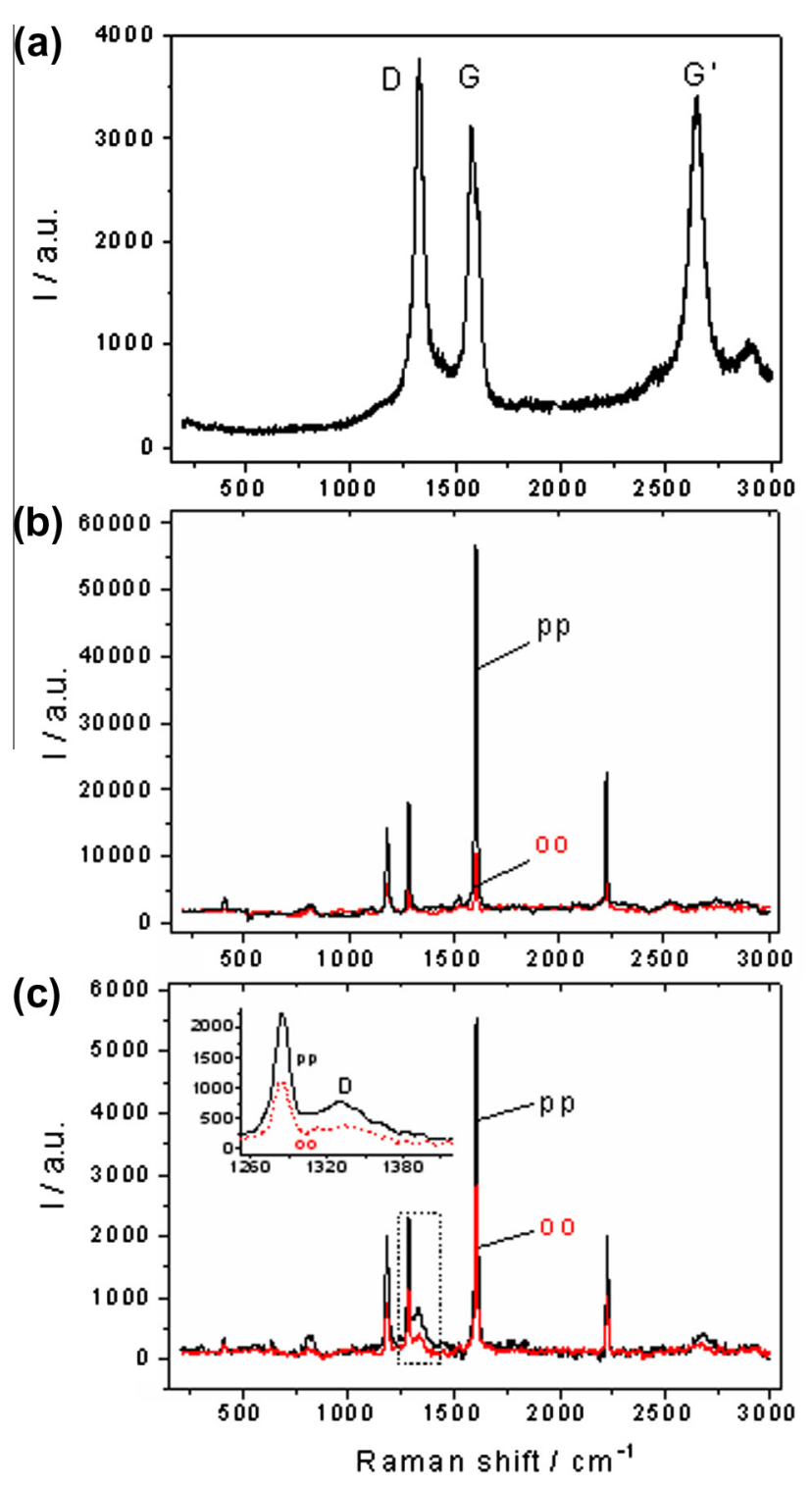

Fig. 6 - Raman spectra of (a) CNTs, (b) LC E7 and (c) E7+CNT (0.1 wt.\%)+LapO (0.1 wt.\%) composite. In cases (b) and (c), the spectra are presented for ee and oo geometry. The inset in Fig. 6c shows magnified part of the spectra marked by rectangle. (A colour version of this figure can be viewed online.)

and/or Lapo particles shows that the dichroism of the LC bands falls with addition of the particles. It indicates some distortion of LC alignment in the vicinity of nanoparticles' aggregates.

\subsection{Electro-optical properties}

Changes in the structure of LC-CNT composites are also reflected in the electro-optical characteristics. Fig. 7 presents transmittance vs. applied voltage curves for pure E7, E7CNT (0.1 wt.\%) and E7-CNT (0.1 wt.\%)-LapO (0.1 wt.\%) samples. Especially clear difference is observed in the saturation values of transmittance. 


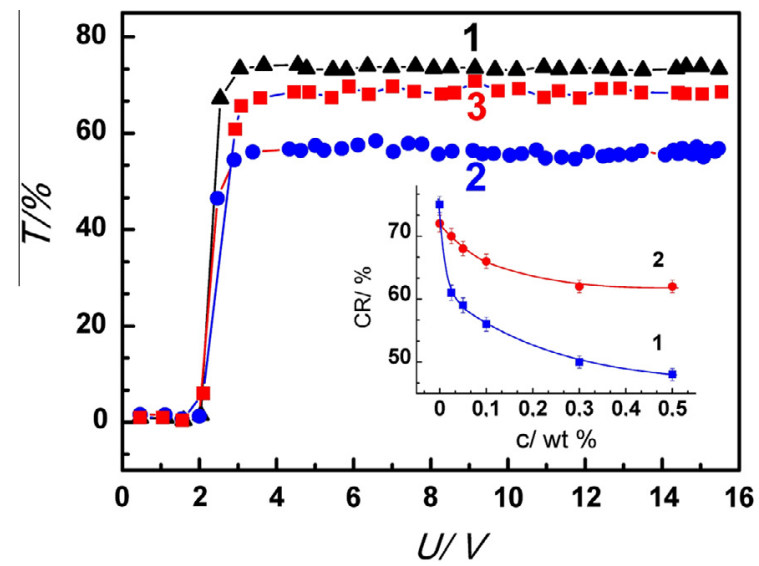

Fig. 7 - Transmittance vs. voltage curves for the E7 (1), E7CNT (0.1 wt.\%) (2) and E7-CNT (0.1 wt.\%)-Lapo (0.1 wt.\%) (3) samples. The inset demonstrates contrast ratio vs. CNT concentration curves for the E7-CNTs (1) and E7-CNTs-LapO (2) series. (A colour version of this figure can be viewed online.)

This value essentially falls down for the E7-CNT sample compared with pure E7, but approaches the E7 value again by adding LapO particles. Accordingly, the switching contrast, considered as a ratio of saturation, $T_{s}$, and initial, $T_{0}$, values of transmittance $T$, is essentially higher for the suspension of CNTs assisted by Laponite. Generally, the change of switching contrast with concentration of CNTs for the LapO-containing series is considerably weaker than for its E7-CNT counterpart (inset to Fig. 7). These features are directly connected to structural changes. The contrast falls down with the addition of CNTs due to absorption of testing light by bulky CNT aggregates.

This effect is much weaker for the LapO-assisted suspensions, because the LapO platelets promote disintegration of CNTs. Individual CNTs and their small aggregates are not such serious obstacles for the testing light as large aggregates in the E7+CNT suspensions. Finally, we detected inessential decrease in Frederiks's threshold $\left(\Delta \mathrm{U}_{\mathrm{F}} \sim 0.1 \mathrm{~V}\right)$ in the LapOcontaining samples. This is apparently due to the influence on the double electric layers of LC cell, through the ion redistribution between the LC and nanoparticles, as in the respective two-component suspensions LC-CNT [12] and LC-clay platelets [50].

\section{Concluding remarks and prospects}

The effect of nanoparticles of organically modified Laponite on dispersion ability of multiwalled carbon nanotubes in a nematic liquid crystal is investigated. It is found that introduction of a small amount (0.1 wt.\%) of LapO platelets in a E7+CNT suspension leads to radical improvement of the dispersion of CNTs in a rather wide range of their concentrations ( $C<0.1 \mathrm{wt}$.\%). Due to intensive exfoliation and dispersion in a LC, as well as high affinity to CNTs, the LapO platelets surround the nanotubes and promote their disaggregation. As a result, only fine aggregates of CNTs are detected in the E7+CNT+LapO suspensions. It is proved that the dispersed nanotubes in these suspensions adopt orientational order of LC matrix.

The enhanced dispersion of CNTs and haloing of them by insulating LapO platelets results in essential changes in dielectric properties of the E7+CNT suspensions. In particular, it eliminates the percolation behavior of conductivity and determines a linear growth of the effective permittivity of the composite with CNT concentration. Furthermore, the LapO-assisted E7+CNT suspensions demonstrate considerably improved electro-optic characteristics, such as better switching contrast and reduced threshold voltage. We believe that the proposed approach is also effective for obtaining highly dispersed states of nanotubes in other types of thermotropic and lyotropic LCs, as well as reactive mesogens, thereby promoting adjustment and diversification of properties of such materials for various optical, electro-optical and electronic applications.

At the same time, it is worthwhile mentioning shortcomings of Laponite as the promoter of dispergation of CNTs in LCs. The major problem is that adding of Laponite to LC leads to essential increase of conductivity. It is not essentially for optical applications, but can be a problem in case of some electro-optical and especially electronic applications. In view of this, our further studies will be focused on the search of other types of nanoparticles, demonstrating high affinity to CNTs and pronounced haloing effect in liquid crystals, in combination with weak effect on the LC conductivity. Recent studies point to several types of particles potentially useful for this purpose [8,51,52].

\section{Acknowledgments}

This work was partially funded under the projects 2.16.1.4, 65/ 13-H (NAS of Ukraine). O.Y. acknowledges support from Fulbright Foundation under the program G-1-00005. Authors thank O. Fesenko for her help in measuring Raman spectra.

\section{Appendix A. Supplementary data}

Supplementary data associated with this article can be found, in the online version, at http://dx.doi.org/10.1016/j.carbon. 2013.11.015.

\section{R E F E R E N C E S}

[1] De Volder MFL, Tawfick SH, Baughman RH, Hart AJ. Carbon nanotubes: present and future commercial applications. Science 2013;339:535-9.

[2] Schnorr JM, Swager TM. Emerging applications of carbon nanotubes. Chem Mater 2011;23:646-57.

[3] Wang X, Liu Y, Zhu D. Controlled growth of well-aligned carbon nanotubes with large diameters. Chem Phys Lett 2001;340(5-6):419-24.

[4] Smith BW, Benes Z, Luzzi DE, Fischer JE, Walters DA, Casavant MJ, et al. Structural anisotropy of magnetically aligned single wall carbon nanotube films. Appl Phys Lett AIP 2000;77(5):663-5.

[5] Hertel T, Martel R, Avouris P. Manipulation of individual carbon nanotubes and their interaction with surfaces. J Phys Chem 1998;B102(6):910-5. 
[6] Zakri C, Poulin P. Phase behavior of nanotube suspensions: from attraction induced percolation to liquid crystalline phases. J Mater Chem 2006;16:4095-8.

[7] Han TH, Kim J, Park JS, Park CB, Ihee H, Kim SO. Liquid crystalline peptide nanowires. Adv Mater 2007;19:3924-7.

[8] Kim JE, Han TH, Lee SH, Kim JY, Ahn CW, Yun JM, et al. Craphene oxide liquid crystals. Angew Chem Int Ed 2011;50:3043-7.

[9] Dierking I, Scalia G, Morales P, LeClere D. Aligning and reorienting carbon nanotubes with nematic liquid crystals. Adv Mater WILEY-VCH Verlag 2004;16(11):865-9.

[10] Lagerwall J, Scalia G. Carbon nanotubes in liquid crystals. J Mater Chem 2008;18(25):2890-8.

[11] Lynch MD, Patrick DL. Organizing carbon nanotubes with liquid crystal solvents. Nano Lett 2002;2(11):1197-201.

[12] Lee W, Wang C-Y, Shih Y-C. Effects of carbon nanosolids on the electro-optical properties of a twisted nematic liquidcrystal host. Appl Phys Lett 2004;85(4):513-5.

[13] Jeong SJ, Park KA, Jeong SH, Jeong HJ, An KH, Nah CW, et al. Electroactive superelongation of carbon nanotube aggregates in liquid crystal medium. Nano Lett 2007;7(8):2178-82.

[14] Baik I-S, Jeon SY, Lee SH, Park KA, Jeong SH, An KH, et al. Electrical-field effect on carbon nanotubes in a twisted nematic liquid crystal cell. Appl Phys Lett AIP 2005;87(26):263110.

[15] Huang C-Y, Hu C-Y, Pan H-C, Lo K-Y. Electrooptical responses of carbon nanotube-doped liquid crystal devices. Jpn J Appl Phys 2005;44:8077-81.

[16] Qi H, Hegmann T. Impact of nanoscale particles and carbon nanotubes on current and future generations of liquid crystal displays. Journal of Materials Chemistry. The Royal Society of. J Mater Chem R Soc Chem 2008;18(28):3288-94.

[17] Schymura S, Kuhnast M, Lutz V, Jagiella S, DettlaffWeglikowska U, Roth S, et al. Towards efficient dispersion of carbon nanotubes in thermotropic liquid crystals. Adv Funct Mater WILEY-VCH Verlag 2010;20(19):3350-7.

[18] Dolgov LA, Lebovka NI, Yaroshchuk OV. Effect of electrooptical memory in suspensions of carbon nanotubes in liquid crystals. Colloid J 2009;71(5):603-11.

[19] Dolgov L, Yaroshchuk O, Tomylko S, Lebovka N. Electrooptical memory of a nematic liquid crystal doped by multiwalled carbon nanotubes. Condens Matter Phys 2012;15(3):33401-8.

[20] Tie W, Yang GH, Bhattacharyya SS, Lee YH. Lee SH. Electric-field-induced dispersion of multiwalled carbon nanotubes in nematic liquid crystal. J Phys Chem C 2011;115(44):21652-8.

[21] Nakashima N. Soluble carbon nanotubes: fundamentals and applications. Int J Nanosci 2005;04(01):119-37.

[22] Kuhnast M, Tschierske C, Lagerwall J. Tailor-designed polyphilic promotors for stabilizing dispersions of carbon nanotubes in liquid crystals. Chem Commun 2010;46:6989-91.

[23] Tohver V, Smay JE, Braem A, Braun PV, Lewis JA. Nanoparticle halos: a new colloid stabilization mechanism. Proceedings of the National Academy of Sciences of the United States of America (PNAS). 2007;104(8):2585-90.

[24] Sun D, Chu C-C, Sue H-J. Simple approach for preparation of epoxy hybrid nanocomposites based on carbon nanotubes and a model clay. Chem Mater 2010;22(12):3773-8.

[25] Loginov M, Lebovka N, Vorobiev E. Laponite assisted dispersion of carbon nanotubes in water. J Colloid Interface Sci 2012;365(1):127-36.

[26] Peeterbroeck S, Alexandre M, Nagy JB, Pirlot C, Fonseca A, Moreau N, et al. Polymer-layered silicate-carbon nanotube nanocomposites: unique nanofiller synergistic effect. Compos Sci Technol 2004;64(15):2317-23.
[27] Liu L, Grunlan JC. Clay assisted dispersion of carbon nanotubes in conductive epoxy nanocomposites. Adv Funct Mater WILEY-VCH Verlag 2007;17(14):2343-8.

[28] Zhang JP, Wang AQ. Synergistic effects of $\mathrm{Na}^{+}$montmorillonite and multi-walled carbon nanotubes on mechanical properties of chitosan film. eXPRESS. Polym Lett 2000;3(5):302-8.

[29] Lysenkov E, Lebovka NI, Yakovlev YV, Klepko VV, Pivovarova NS. Percolation behaviour of polypropylene glycol filled with multiwalled carbon nanotubes and Laponite. Compos Sci Technol 2012;72(10):1191-5.

[30] Lebovka N, Goncharuk A, Bezrodna T, Chashechnikova I, Nesprava V. Microstructure and electrical conductivity of hybrid liquid crystalline composites including 5CB, carbon nanotubes and clay platelets. Liq Cryst 2012;39(5):531-8.

[31] Melezhyk AV, Sementsov YuI, Yanchenko VV. Synthesis of porous carbon nanofibers on catalysts fabricated by the mechanochemical method. Russuan. J Appl Chem 2005;78(6):924-30.

[32] Zebrowski J, Prasad V, Zhang W, Walker LM, Weitz DA. Shakegels: shear-induced gelation of laponite-PEO mixtures. Colloids Surf A Physicochem Eng Aspects 2003;213(23):189-97.

[33] Hoshen J, Kopelman R. Percolation and cluster distribution. I. Cluster multiple labeling technique and critical concentration algorithm. Phys Rev B 1976;14(8):3438-45.

[34] Twarowski AJ, Albrecht AC. Depletion layer studies in organic films: low frequency capacitance measurements in polycrystalline tetracene. J Chem Phys 1979;70:2255-61.

[35] Dolgov L, Kovalchuk O, Lebovka N, Tomylko S, Yaroshchuk O. Liquid crystal dispersions of carbon nanotubes: Dielectric, electro-optical and structural peculiarities. In: Marulanda JM, editor. Carbon nanotubes. InTech; 2010. p. 451-84.

[36] Blinov L. Structure and Properties of Liquid Crystals. Dordrecht: Springer; 2011.

[37] Bezrodna T, Chashechnikova I, Dolgov L, Puchkovska G, Shaydyuk Y, Lebovka N, et al. Effects of montmorillonite modification on optical properties of heterogeneous nematic liquid crystal-clay mineral nanocomposites. Liq Cryst 2005;32(8):1005-12.

[38] Van Duijneveldt JS, Klein S, Leach E, Pizzey C, Richardson RM. Large scale structures in liquid crystal/clay colloids. J Phys Condens Matter 2005;17(15):2255.

[39] Lisetski LN, Minenko SS, Ponevchinsky VV, Soskin MS, Goncharuk AI, Lebovka NI. Microstructure and incubation processes in composite liquid crystalline material (5CB) filled with multi walled carbon nanotubes. Materialwiss Werkstofftech WILEY-VCH Verlag 2011;42(1):5-14.

[40] Stauffer D, Aharony A. Introduction to Percolation Theory. second ed. CRC Press; 1994.

[41] Lebovka NI, Manna SS, Tarafdar S, Teslenko N. Percolation in models of thin film depositions. Phys Rev E Am Phys Soc 2002;66(6):66134.

[42] Müller K-H, Wei G, Raguse B, Myers J. Three-dimensional percolation effect on electrical conductivity in films of metal nanoparticles linked by organic molecules. Phys Rev B Am Phys Soc 2003;68(15):155407.

[43] Hensen E, Smit B. Why clays swell. J Phys Chem B 2002;106:12664-7.

[44] Huang Y, Bhowmik A, Bos P. Characterization of ionic empurities adsorbed onto a $5^{\circ} \mathrm{SiO}_{x}$ alignment film. Jpn J Appl Phys 2012;51:031701.

[45] Basu R, Iannacchione GS. Orientational coupling enhancement in a carbon nanotube dispersed liquid crystal. Phys Rev E 2010;81(5):51705.

[46] Choy TC. Effective Medium Theory. Oxford: Clarendon Press; 1999. 
[47] Scalia G, Haluska M, Dettlaff-Weglikowska U, Giesselmann F, Roth S. Polarized Raman spectroscopy study of SWCNT orientational order in an aligning liquid crystalline matrix. AIP Conf Proc 2005;786:114.

[48] Scalia G, von Buehler C, Haegele C, Roth S, Giesselmann F, Lagerwall JPF. Spontaneous macroscopic carbon nanotube alignment via colloidal suspension in hexagonal columnar lyotropic liquid crystals. Soft Matter 2008;4:570.

[49] Dresselhaus MS, Dresselhaus G, Saito R, Jorio AR. Raman spectroscopy of carbon nanotubes. Phys Rep 2005;409(2):47.
[50] Tsai T-Y, Huang Y-P, Chen H-Y, Lee W, Chang Y-M, Chin W-K. Electro-optical properties of a twisted nematicmontmorillonite-clay nanocomposite. Nanotechnology 2005;16(8):1053.

[51] Wu P-C, Lee W. Phase and dielectric behaviors of polymerphic liquid crystals doped with graphene nanoplatelets. Appl Phys Lett 2013;102:162904.

[52] Kyrylyuk AV, Hermant MC, Schilling T, Klumperman B, Konig CE. Controlling electrical percolation in multicomponent carbon nanotube dispersion. Nat Nanotechnol 2011;6:364-9. 